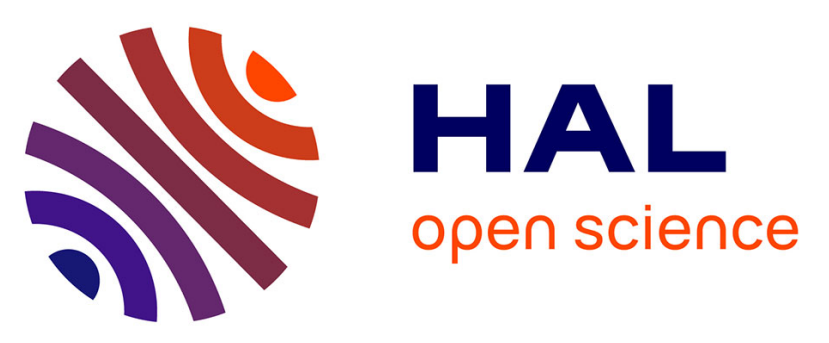

\title{
Investigation of the spatial distribution of hot carriers in quantum-well structures via hyperspectral luminescence imaging
}

\author{
Hamidreza Esmaielpour, Laurent Lombez, Maxime Giteau, Amaury \\ Delamarre, Daniel Ory, Andrea Cattoni, Stéphane Collin, Jean-François \\ Guillemoles, Daniel Suchet
}

\section{To cite this version:}

Hamidreza Esmaielpour, Laurent Lombez, Maxime Giteau, Amaury Delamarre, Daniel Ory, et al.. Investigation of the spatial distribution of hot carriers in quantum-well structures via hyperspectral luminescence imaging. Journal of Applied Physics, 2020, 128 (16), pp.165704. 10.1063/5.0022277 . hal-02988730

\section{HAL Id: hal-02988730 \\ https://hal.science/hal-02988730}

Submitted on 4 Nov 2020

HAL is a multi-disciplinary open access archive for the deposit and dissemination of scientific research documents, whether they are published or not. The documents may come from teaching and research institutions in France or abroad, or from public or private research centers.
L'archive ouverte pluridisciplinaire HAL, est destinée au dépôt et à la diffusion de documents scientifiques de niveau recherche, publiés ou non, émanant des établissements d'enseignement et de recherche français ou étrangers, des laboratoires publics ou privés. 


\section{Investigation of the spatial distribution of hot carriers in quantum-well structures via hyperspectral luminescence imaging}

Cite as: J. Appl. Phys. 128, 165704 (2020); https://doi.org/10.1063/5.0022277

Submitted: 20 July 2020 . Accepted: 13 October 2020 . Published Online: 30 October 2020

Hamidreza Esmaielpour (iD), Laurent Lombez (D), Maxime Giteau (D), Amaury Delamarre (iD), Daniel Ory (D), Andrea Cattoni $(\mathbb{D}$, Stéphane Collin $(\mathbb{D}$, Jean-François Guillemoles $\mathbb{D}$, and Daniel Suchet $(\mathbb{D})$
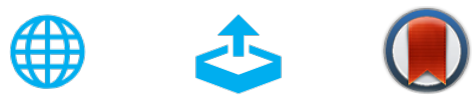

View Online

Export Citation

\section{ARTICLES YOU MAY BE INTERESTED IN}

Analytical description of mixed ohmic and space-charge-limited conduction in single-carrier devices

Journal of Applied Physics 128, 165701 (2020); https://doi.org/10.1063/5.0024737

Dynamics of surface electron trapping of a GaN-based transistors revealed by spatiotemporally resolved $\mathrm{x}$-ray spectroscopy

Applied Physics Letters 117, 171605 (2020); https://doi.org/10.1063/5.0020500

Realization of high thermoelectric power factor in Ta-doped ZnO by grain boundary engineering

Journal of Applied Physics 128, 165110 (2020); https://doi.org/10.1063/5.0022287

\section{Meet the Next Generation of Quantum Analyzers And Join the Launch Event on November 17th}

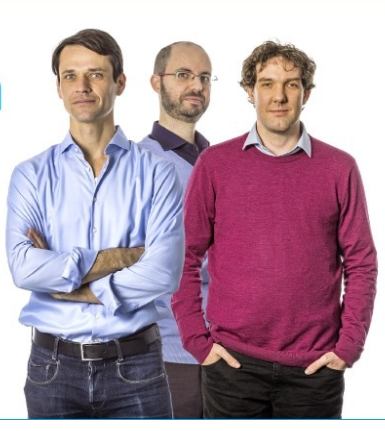

\section{Register now}

Zurich Instruments 


\title{
Investigation of the spatial distribution of hot carriers in quantum-well structures via hyperspectral luminescence imaging
}

Cite as: J. Appl. Phys. 128, 165704 (2020); doi: 10.1063/5.0022277

Submitted: 20 July 2020 . Accepted: 13 October 2020 .

Published Online: 30 October 2020

Hamidreza Esmaielpour, ${ }^{1, a)}$ (D) Laurent Lombez, ${ }^{1,2}$ (i) Maxime Giteau, ${ }^{3}$ (iD) Amaury Delamarre, ${ }^{3,4}$ (D) Daniel Ory, ${ }^{1,5}$ (D) Andrea Cattoni, ${ }^{1,4}$ (iD) Stéphane Collin, ${ }^{1,4}$ (D) Jean-François Guillemoles, ${ }^{1,6}$ (D) and Daniel Suchet ${ }^{3,6, a)}$ (D)

\author{
AFFILIATIONS \\ ${ }^{1}$ Institute Photovoltaïque d'lle de France (IPVF), UMR IPVF 9006, Palaiseau, France \\ ${ }^{2}$ Laboratoire de Physique et Chimie des Nano-objets (LPCNO-INSA), Toulouse, France \\ ${ }^{3}$ NextPV, LIA, Research Center for Advanced Science and Technology (RCAST), University of Tokyo, Tokyo, Japan \\ ${ }^{4}$ Centre de Nanosciences et de Nanotechnologies (C2N), CNRS, Université Paris-Saclay, Palaiseau, France \\ ${ }^{5}$ Électricité de France (EDF) R\&D, Palaiseau, France \\ ${ }^{6}$ CNRS-Ecole Polytechnique, UMR IPVF 9006, Palaiseau, France
}

Note: This paper is part of the Special Topic on Hot Electron Physics and Applications.

${ }^{a}$ Authors to whom correspondence should be addressed: hamidreza.esmaielpour@cnrs.fr and daniel.suchet@polytechnique.org

\begin{abstract}
Observation of robust hot carrier effects in quantum-well structures has prompted hopes to increase the efficiency of solar cells beyond the Shockley-Queisser limit (33\% for single junction solar cells at AM1.5G). One of the main studies in hot carrier effects is the determination of carrier temperature, which provides information on the thermalization mechanisms of hot carriers in semiconductor materials. Here, we investigate the spatial distribution of photo-generated hot carriers in a InGaAs multi-quantum-well structure via hyperspectral luminescence imaging. We discuss proper methods of extracting the temperature of carriers from a photoluminescence spectrum. Robust hot carrier effects are observed at the center of the laser spot at various lattice temperatures. In addition, it is seen that the local carrier temperature scales linearly with the local laser intensity as long as the illumination exceeds a threshold power; the carrier temperature at regions with local intensities below the threshold drops to the lattice temperature, i.e., experiences no hot carrier effects. Moreover, at large distances from the concentrated light, where the level of illumination is negligible, evidence of carrier radiative recombination is observed, which is attributed to carrier diffusion in the planar structure. The results of this study can be applied to investigate the influence of carrier diffusion and thermoelectric effects on the thermalization of hot carriers.
\end{abstract}

Published under license by AIP Publishing. https://doi.org/10.1063/5.0022277

\section{INTRODUCTION}

Extraction of photo-generated hot carriers in single junction solar cells can boost the efficiency of these devices beyond their fundamental limit (33\%, known as the Shockley-Queisser limit). ${ }^{1}$ This mechanism is the basis for hot carrier solar cell technology, which offers an efficiency enhancement up to $\sim 67 \%$ under one sun illumination for single junction photovoltaic devices. ${ }^{1}$

Absorption of high energy photons, larger than semiconductor bandgaps, creates electron-hole pairs with temperatures larger than those of the crystal lattice. This process is followed by a fast relaxation of hot carriers to the band edge of the semiconductor, known as a thermalization mechanism. ${ }^{1-3}$ In most types of semiconductors, this process occurs in the order of a few picoseconds (ps), and the energy loss due to heat dissipation in thermalization mechanisms limits the power conversion efficiency of solar cells. ${ }^{3}$ However, it is possible to harness the excess kinetic energy of hot carriers and to improve the efficiency of solar cells by (1) reducing the rate of thermalization and (2) extracting the hot carriers quickly before they thermalize. ${ }^{4,5}$ Different approaches, such as nanostructured materials, ${ }^{6-10}$ phononic engineering, ${ }^{11-13}$ and intervalley 
scattering, ${ }^{14,15}$ have been proposed to reduce the thermalization rate of hot carriers and to increase the efficiency of hot carrier solar cells.

Determining the temperature of carriers in semiconductors provides rich information about hot carriers in these materials. Experimentally, the temperature of hot carriers has been determined using various experiments, such as ultrafast pump-probe spectroscopy, ${ }^{16-18}$ spontaneous photoemission spectroscopy, ${ }^{19}$ and electrical measurements. ${ }^{20,21}$ One widely used technique is steady state photoluminescence (PL) spectroscopy, which can determine the optical properties of semiconductors, as well as the thermodynamic properties of emitting particles, such as temperatures and quasi-Fermi level splitting. ${ }^{22-26}$ By fitting a PL spectrum with the generalized Planck's law, the temperature of emitting particles can be found. In this analysis, it is required to have information about the absorptivity of the sample, which is an energy dependent parameter. In one widely used technique, the carrier temperature is determined in the range of energies where the absorptivity is constant, known as the linear fitting method. ${ }^{2}$ However, if the absorptivity changes above the bandgap energy, which is the case for nanostructured materials, the temperature extracted by the linear fitting may be inaccurate. ${ }^{27}$

There are important questions about hot carrier effects in quantum-well (QW) structures, such as how they depend on lattice temperature and excitation power. In this work, we report experimental investigations of these questions based on hyperspectral luminescence imaging, which allows for an effective power-dependent measurement in a single shot. Through these experiments, spatial and energy distributions of hot carriers in a InGaAs multiquantum-well (MQW) structure are investigated. The schematic of the hyperspectral system and an image of PL emission by the MQW structure are shown in Figs. 1(a) and 1(b), respectively. With hyperspectral imaging, it is possible to have access to PL spectra emitted by different regions of the sample and, therefore, to determine the temperature of hot carriers across the sample. Emitted PL spectra by the InGaAs MQW structure at various distances from the center of the laser spot are plotted in Fig. 1(c). The graph indicates that there is a change in the PL spectra with the distance from the center of the concentrated laser $(\Delta x=0 \mu \mathrm{m})$. In Sec. II, we present fitting methods to accurately determine the temperature of carriers from the PL spectrum. In Sec. III, we discuss how photo-generated hot carriers are distributed spatially across the MQW structure. In addition, the correlation between local laser excitation and the temperature of hot carriers in the sample is discussed. Finally, we discuss how the threshold power for the creation of a stabilized hot carrier distribution can be determined by hyperspectral luminescence imaging.

\section{DETERMINATION OF THE CARRIER TEMPERATURE}

PL emission in semiconductors can be applied to determine the temperature " $T$ " and the quasi-Fermi level splitting " $\Delta \mu$ " of emitting particles. These parameters are found by fitting an emitted PL spectrum with the generalized Planck's law. This equation is described by $^{2,24,26}$

$$
I_{P L}(E)=\frac{2 \pi A(E) E^{2}}{h^{3} c^{2}}\left[\exp \left(\frac{E-\Delta \mu}{k_{B} T}\right)-1\right]^{-1},
$$
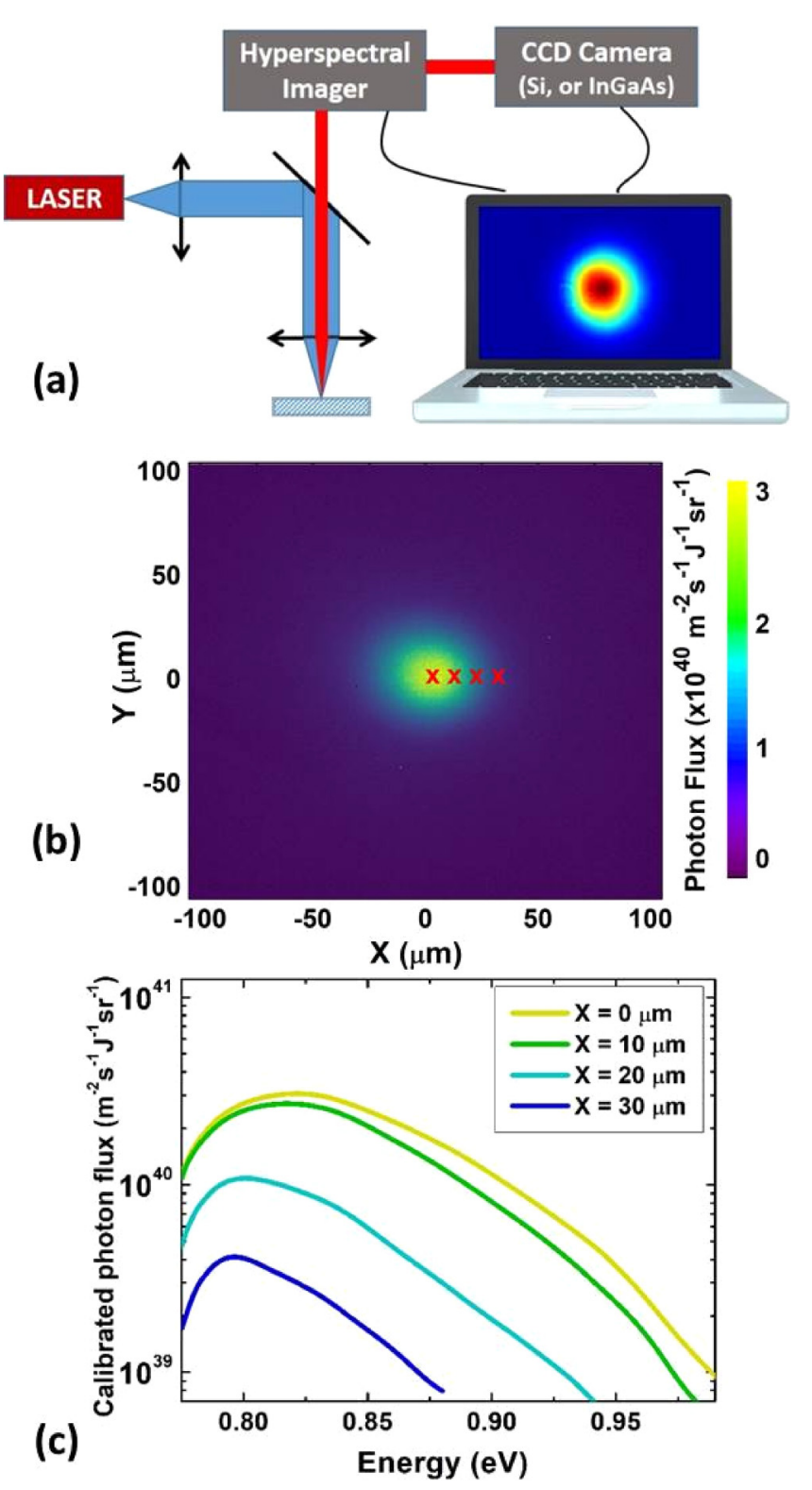

FIG. 1. (a) Schematic of the hyperspectral imager setup. (b) PL map of the InGaAs MQW structure at $300 \mathrm{~K}$ registered by the hyperspectral imager. The red marks show the selected regions where their PL spectra are plotted in the next panel. (c) PL spectra at various distances from the center of the laser (or $\mathrm{PL}$ ) spot, taken from the PL map in panel (b).

where " $I_{P L}$ " is the PL intensity, " $A$ " the absorptivity of the sample, " $h$ " Planck's constant, " $k_{B}$ " Boltzmann's constant, and " $c$ " the speed of light. After photo-absorption in semiconductors, electrons and holes in the conduction and valence bands, respectively, distribute energy among each other via carrier-carrier scattering and achieve a state of quasi-thermal equilibrium (or non-equilibrium). ${ }^{28}$ 
Therefore, carriers in each band are at thermal equilibrium but not with carriers in the other band and the lattice. This effect is due to the fast carrier-carrier scattering relative to carrier-phonon interactions and carrier recombination. ${ }^{3}$ In this condition, there are two distinct Fermi levels and temperatures associated with electrons and holes in the system, which define their thermodynamic properties in each band. ${ }^{28,29}$ In Eq. (1), $\Delta \mu$ refers to the energy separation between the quasi-Fermi levels of electrons and holes in the semiconductor and $T$ is the average temperature of electrons and holes.

The simulation result of Eq. (1) for a semiconductor with a bandgap energy of $0.78 \mathrm{eV}$ is plotted in Fig. 2(a). In this simulation, only radiative recombination associated with one band-to-band transition (from the bottom of the conduction band to the top of the valence band) is considered. As shown in the semi-logarithmic graph of Fig. 2(a), the PL intensity increases with the photon energy up to the bandgap of the semiconductor and then decreases linearly.

As indicated in Eq. (1), there are three energy-dependent terms in the generalized Planck's law, and their behaviors as a function of energy are plotted in Fig. 2(b). It is observed that the natural logarithm of the absorptivity and the exponential term, the exponent of which is inversely proportional to the carrier temperature, has large contributions below and above the bandgap energy $(0.78 \mathrm{eV})$, respectively. In addition, the contribution of "ln $\left(E^{2}\right)$ " is large only at low energy values; however, its variation becomes negligible at higher energy values $(E>0.7 \mathrm{eV})$. Figure $2(\mathrm{~b})$ indicates that it is valid to estimate the carrier temperature by applying a linear fitting at energy ranges far from the bandgap where the absorptivity remains relatively constant. This simple analysis is a common technique for the determination of the carrier temperature. However, this analysis can make a good estimate of the carrier temperature only if the linear fit is applied in the energy range where the absorptivity is constant.

To investigate hot carrier effects in the InGaAs MQW structure, continuous wave PL spectroscopy is performed. The InP lattice-matched MQW structure consists of five un-doped $\mathrm{In}_{0.53} \mathrm{Ga}_{0.47}$ As QWs (5.5 nm thick) and $\mathrm{In}_{0.8} \mathrm{Ga}_{0.2} \mathrm{As}_{0.44} \mathrm{P}_{0.56}$ barriers $(10 \mathrm{~nm}$ thick). InP cladding layers are grown at the top and bottom of the MQW structure to accumulate photo-generated carriers within the active region. The schematic and the band energy diagram of the sample are shown in Figs. 3(a) and 3(b), respectively. The red dotted lines within the QWs indicate the positions of discrete energy levels created by quantum confinement effects.

Figure 4(a) shows PL spectra emitted by the MQW structure at $300 \mathrm{~K}$ under various excitation powers of a $405 \mathrm{~nm}$ laser diode. In this measurement, the energy of incident photons on the sample is larger than the bandgap energy of the QW $(0.78 \mathrm{eV})$ and the barrier $(1.13 \mathrm{eV})$ materials. Due to the existence of discrete energy levels within the MQW structure, multiple features associated with the occupation of excited states are observed at the high energy side of the PL spectra; see Fig. 4(a). This effect is more evident when the energy separation of the quantized levels is smaller than $<k_{B} T_{L}$ ( $T_{L}$ is the lattice temperature). As a result, when photogenerated carriers receive enough thermal energy (under high illumination and/or at elevated lattice temperatures), the probability of occupation of the excited states becomes larger and, consequently, stronger PL emission is observed from these states. This effect
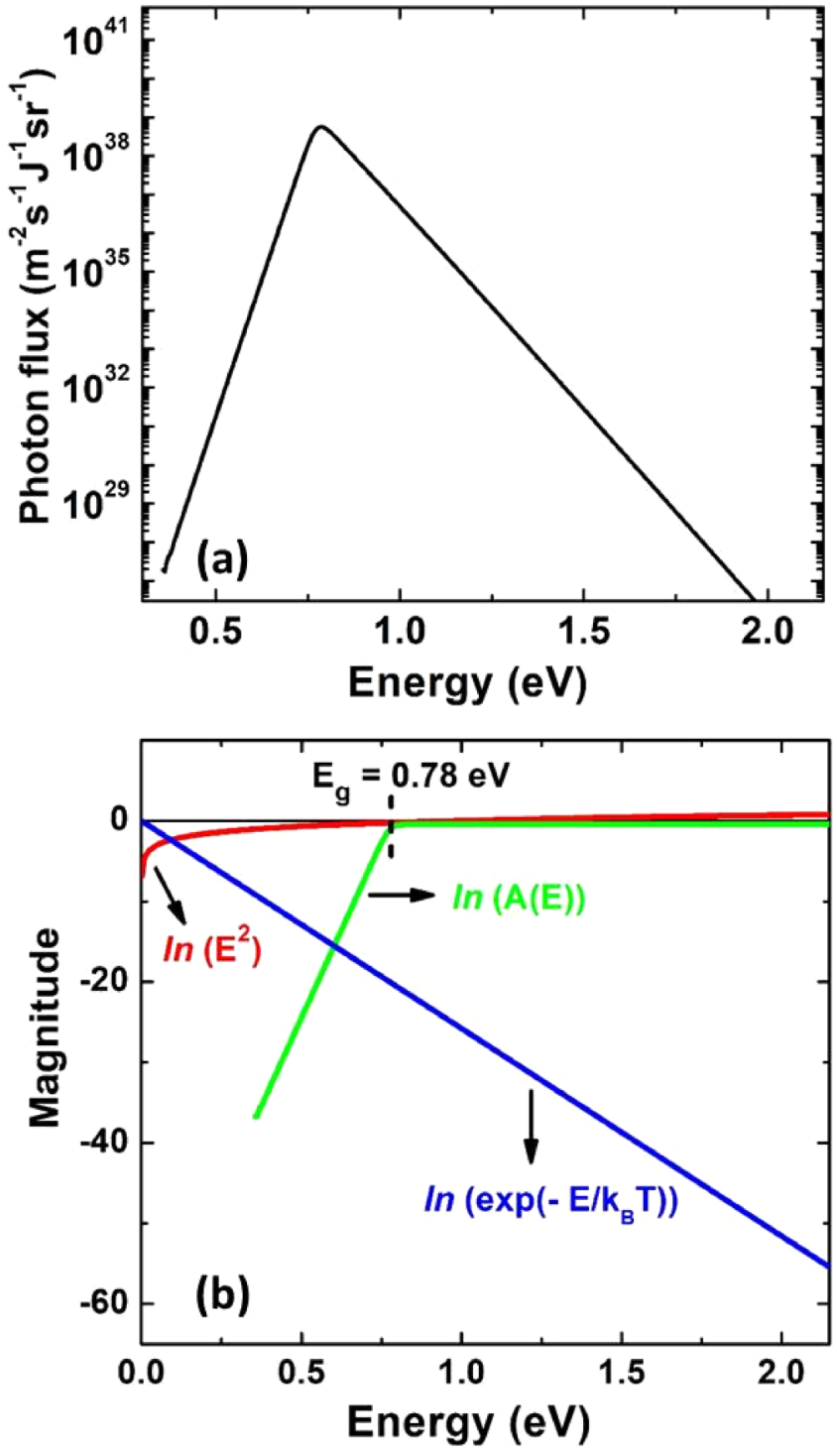

FIG. 2. (a) Theoretical simulation of a PL spectrum emitted by a semiconductor with a bandgap energy of $0.78 \mathrm{eV}$. (b) The contribution of energy-dependent terms in the generalized Planck's equation.

changes the shape of PL spectra and adds complications to the carrier temperature determination.

The red dashed lines in Fig. 4(a) indicate the range of energies where the carrier temperatures are found by the linear fitting method. The determination of the carrier temperature in nanostructured materials by this common technique can be challenging and may lead to erroneous results. ${ }^{27,30,31}$ In addition, depending upon the range of energies to which the linear fitting is applied, extracted carrier temperatures will be different. This effect is associated with their absorptivity, which has an energy-dependent behavior above the bandgap. Therefore, the extraction of the carrier 


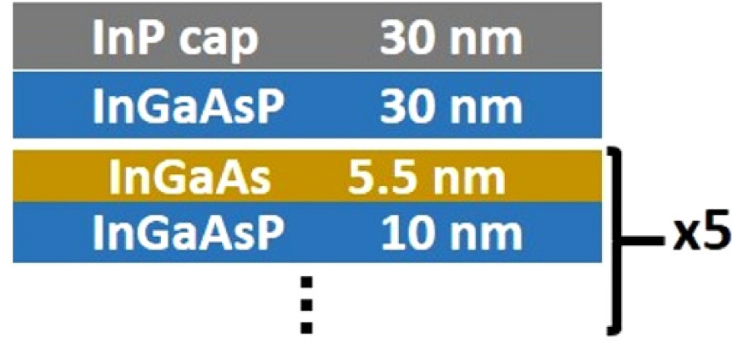

\section{InGaAsP $\quad 20 \mathrm{~nm}$}

(a) InP Substrate

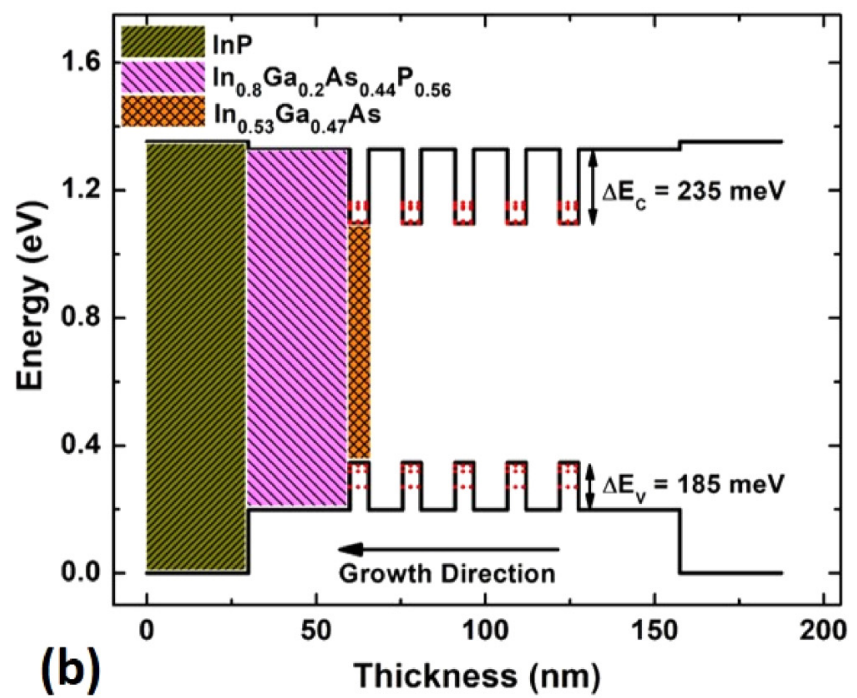

FIG. 3. (a) Schematic and (b) the band energy diagram of the InGaAs MQW structure at $300 \mathrm{~K}$.

temperature in these materials requires taking into account their absorptivity and multiple optical transitions from their quantized energy levels.

The second method of determining the carrier temperature, which is more accurate than the linear fitting technique, is to fit the full PL spectrum with Eq. (1). This method requires to know the optical transitions in the system and to build an absorptivity model " $A(E)$." This comprehensive fitting method provides a more accurate estimate of the temperature and the quasi-Fermi level splitting of carriers in the system. ${ }^{8,27}$ In addition, in the full fitting of the PL spectrum, it is possible to include the band filling effect, which occurs under high illumination, and to determine separate temperatures for electrons and holes. ${ }^{8,26}$

At intense excitation powers, when the quasi-Fermi level splitting is equal to or greater than the bandgap, $\left(\Delta \mu \geq \varepsilon_{g}\right)$, available states at the band edge of the semiconductor become saturated and then carriers occupy higher energy levels within the band. ${ }^{32,33}$ In this condition, the absorption of the system shifts to higher energy values as a function of the absorbed power, known as band filling or the Moss-Burstein effect. ${ }^{34,35}$ Therefore, in order to determine the thermodynamic properties of the emitting particles from their PL spectrum, it is required to fit the entire PL spectrum by solving the Fermi-Dirac integral in Eq. (1). ${ }^{36}$

In the full fitting of the PL spectrum, the contribution of optical transitions from discrete energy levels is included in the absorptivity model, as given by ${ }^{26,37}$

$$
A(E)=[1-R(E)] \cdot\left[1-\exp \left(-\left[\alpha_{w} d_{w}+\alpha_{b} d_{b}\right]\right)\right],
$$

where " $R$ " is reflectivity, " $d$ " the absorber thickness, and " $\alpha$ " the absorption coefficient of the sample. The indices " $w$ " and " $b$ " represent the QW and the barrier materials, respectively. The accurate description of the absorptivity can imbed several features, which are included in its absorption coefficients, such as the energy, the linewidth, and the amplitude of optical transitions. ${ }^{26,37}$ The absorption coefficient " $\alpha_{w 0}$ " of the MQW structure is determined by $8,37,38$

$$
\begin{aligned}
\alpha_{w 0}(E)= & a_{x} \cdot \exp \left[-\frac{\left(E-E_{x}\right)^{2}}{2 \Gamma_{x}^{2}}\right] \\
& +\sum_{i=1}^{n} a_{i} \cdot \frac{1}{1+\exp \left(-\frac{E-E_{i}}{\Gamma_{i}}\right)} \cdot \frac{2}{1+\exp \left(-2 \pi \sqrt{\frac{R_{y}}{\left|E-E_{i}\right|}}\right)},
\end{aligned}
$$

and the absorption coefficient " $\alpha_{b 0}$ " of the barrier material is

$$
\alpha_{b 0}(E)=a_{b} \cdot \frac{1}{1+\exp \left(-\frac{E-E_{b}}{\Gamma_{b}}\right)},
$$

where " $a$," " $E$," and " $\Gamma$ " are the amplitude, the energy, and the linewidth broadening of the absorption coefficient, respectively. " $R_{y}$ " is the effective Rydberg energy of the material, i.e., the exciton binding energy $\left(E_{i}=R_{y}+E_{x}\right)$. The first term in Eq. (3), represented by the index " $x$," describes an optical transition due to excitons in the QW structure, which has a large contribution around the quantized energy levels. The second term in Eq. (3) refers to multiple $(i=1, \ldots, n)$ band-to-band radiative recombination mechanisms from discrete energy levels in the QW structure multiplied by a Sommerfeld factor (associated with Coulomb interactions between electrons and holes). ${ }^{39}$ Here, the absorption coefficient of the InGaAs MQW is modeled by one excitonic and three $(i=1,2,3)$ band-to-band transitions (the Sommerfeld factor is only considered for the first transition, $i=1$, to simplify the analysis). The band filling effect in the MQW structure is described by 8,40

$$
\frac{\alpha}{\alpha_{0}}=f_{v}^{e}-f_{c}^{e}=\frac{\sinh \left(\frac{E-\Delta \mu}{2 k_{B} T}\right)}{\cosh \left(\frac{E-\Delta \mu}{2 k_{B} T}\right)+\cosh \left[\frac{m_{h}-m_{e}}{m_{h}+m_{e}} \cdot \frac{E-E_{g}}{2 k_{B} T}-\frac{D}{4} \ln \left(\frac{m_{h}}{m_{e}}\right)\right]},
$$




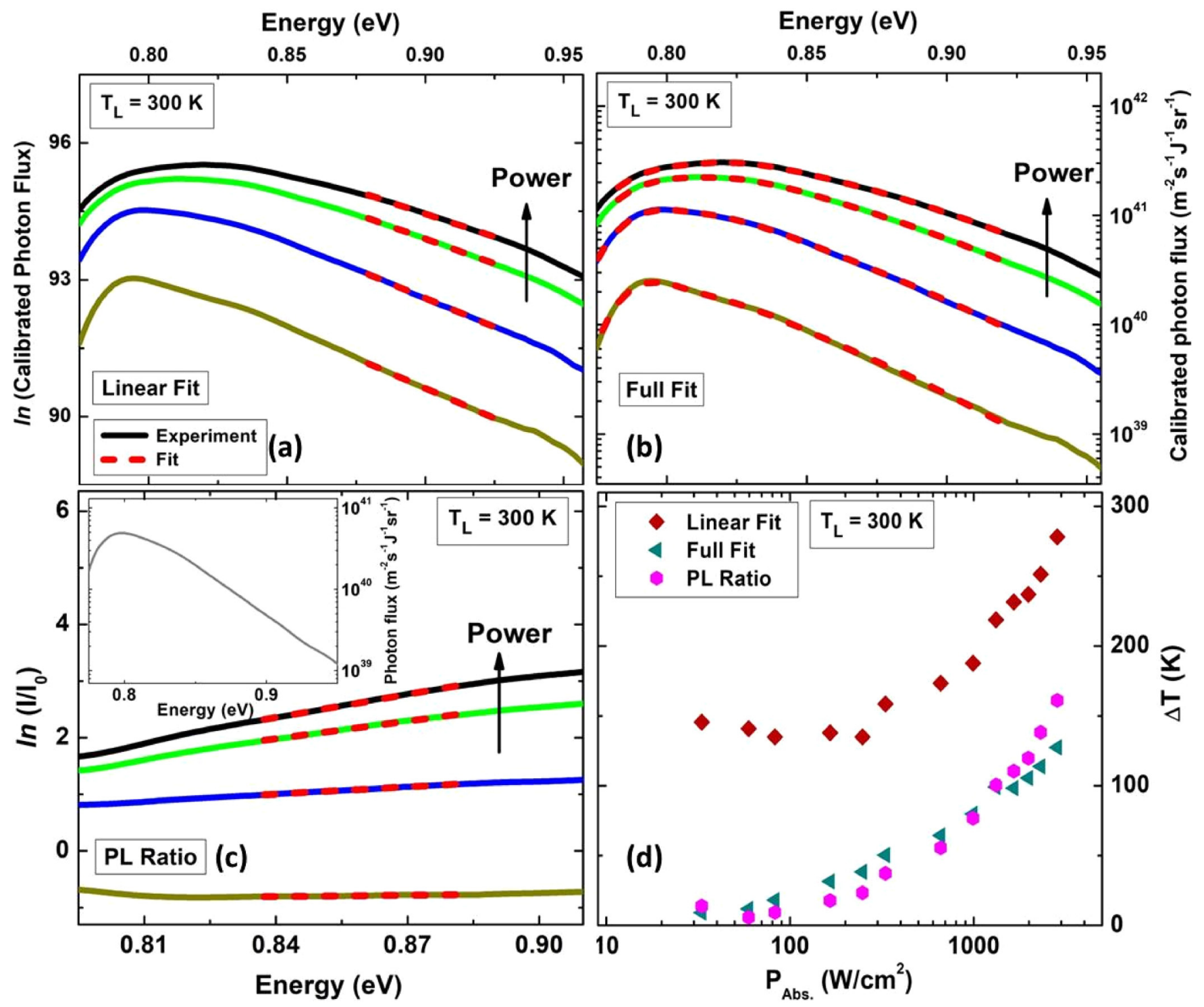

FIG. 4. PL spectra at $300 \mathrm{~K}$ under various intensities analyzed by (a) the linear fitting, (b) the full PL spectrum fitting, and (c) the PL ratio method. The inset of figure (c) shows the reference PL spectrum emitted from a region $15 \mu \mathrm{m}$ from the laser spot under low excitation. (d) The comparison of the temperature difference $(\Delta T)$ determined from the three methods vs the absorbed power density.

where " $f_{v}^{e " ~ a n d ~ " ~} f_{c}^{e}$ " are the Fermi-Dirac distributions of electrons in the valence band and the conduction band, respectively. " $D$ " refers to the dimensionality of the system $(D=2$ for QW structures and $D=3$ for bulk semiconductors). " $m_{h}$ " and " $m_{e}$ " in Eq. (6) are the effective mass of holes and electrons, respectively.

Figure 4(b) shows the results of the full PL fitting on the same PL spectra as Fig. 4(a). Although there are multiple (17) variables in the absorptivity model [Eqs. (2)-(6)] and the generalized Planck's law [Eq. (1)], it is possible to identify them by fitting the equations with PL spectra emitted at various excitation powers. It is seen that there is a good agreement between the experimental and the fitting results; see Fig. 4(b). Through this fitting, it is possible to determine the temperature and the quasi-Fermi level splitting of hot carriers in the MQW structure.

Although the full fitting method improves the accuracy of the results, its analysis can be slow and complicated depending upon the nanostructured design. In addition, this technique requires calibration methods in order to determine the absolute number of photon flux emitted by the sample (intensity calibration) and to 
remove the optical artifacts imposed by optical characterization devices (spectral calibration).

Another approach for determining the temperature of carriers in semiconductors is to find the ratio between two emitted PL spectra, which have the same absorptivity. With this technique, it is possible to remove the absorptivity term (as well as $E^{2}$ ) and to avoid its complexity in the analysis. The PL ratio method is described by

$$
\ln \left(\frac{I_{n}}{I_{r e f}}(E)\right) \approx\left[\frac{\mu_{n}}{k_{B} T_{n}}-\frac{\mu_{r e f}}{k_{B} T_{r e f}}\right]-\left[\frac{1}{k_{B} T_{n}}-\frac{1}{k_{B} T_{r e f}}\right] \times E,
$$

where the index "ref" is associated with a reference PL spectrum with a well-defined carrier temperature and the index " $n$ " refers to the PL spectrum with an unknown " $T$ " and " $\mu$." This analysis is valid under the Boltzmann approximation where the exponential term in Eq. (1) is much larger than unity $\left[\exp \left(E-\Delta \mu / k_{B} T\right) \gg 1\right]$. It is possible to verify the validation of this approximation via determining $n / N_{C}$ and $p / N_{V}$ whose values must be less than unity $(<1)$. Here," $n$ " and " $p$ " are the density of photo-generated electrons and holes in the semiconductor, and " $N_{C}$ " and " $N_{V}$ " are effective density states in the conduction and valence bands, respectively. Equivalently, this condition implies that the density of photogenerated electrons (and holes) is much smaller than the effective density of states in the conduction band (and in the valence band). In addition, the absorptivity of both PL spectra must be the same, which is the case when there is no change in the lattice temperature and the band filling effect is negligible.

The PL ratio method is more accurate than the linear fitting and less complex compared with the full fitting of the PL spectrum. Therefore, it is possible to apply this technique for nanostructured materials with discrete energy levels. In addition, this analysis does not require any calibration procedures; optical artifacts and/or parasitic absorptions imposed by optical characterization devices are eliminated by taking the ratio of the PL spectra.

The PL ratio method, however, requires a reference PL spectrum with a well-defined carrier temperature. Determining a correct reference spectrum is essential in this technique because it influences both the absolute value and the gradient of the change in the carrier temperature. One way to find a reference spectrum is to excite the sample under a low illumination light whose intensity is below the threshold power for the creation of a stabilized hot carrier distribution. Therefore, the temperature of emitting particles remains the same as the lattice temperature. One problem with this method is the low signal-to-noise ratio of the registered PL spectrum, which may add uncertainties to the results. Another technique is to find a reference spectrum via hyperspectral images. As shown in Fig. 1(b), it is possible to have access to PL spectra in regions far from the center of the laser spot (larger than the diffusion lengths of hot carriers $\left.{ }^{41}\right)$. Therefore, particles in these regions are in thermal equilibrium with the lattice $(\Delta T=0 \mathrm{~K}$; the temperature difference between carriers and the lattice) and their emitted PL spectra have a sufficient signal-to-noise ratio for data analysis.

The results of the PL ratio method applied on the PL spectra of the InGaAs MQW structure are shown in Fig. 4(c). The inset of this graph indicates the reference PL spectrum considered for the analysis. The reference PL spectrum is registered by the hyperspectral luminescence imager, and it is emitted from a region $15 \mu \mathrm{m}$ away from the center of the laser spot with a low excitation power. The solid pink lines in Fig. 4(c) indicate the range of energies where the slopes are extracted. The carrier temperatures are calculated by

$$
T_{n}=\frac{T_{r e f}}{1-k_{B} T_{r e f} \times(\text { slope })} .
$$

The results of the power-dependent carrier temperature, determined by various fitting methods, are plotted in Fig. 4(d). It is seen that the linear fitting method indicates higher values for the carrier temperature than the other methods (almost twice as much as them). In addition, $\Delta T$ never reaches zero, even at low excitation powers $(\Delta T=150 \mathrm{~K}$ at the lowest intensity!). This effect indicates that there are always stabilized hot carrier distributions in the sample regardless of the level of illumination. This result cannot be correct because at this condition, the excitation power is not sufficient to create such a robust hot carrier distribution. This is an example of how the common method (linear fitting) to extract the carrier temperature can give erroneous results.

The results of the carrier temperature determined by the PL ratio and the full fitting are in good agreement with each other, and their differences can come from the assumptions applied for the absorptivity model. One source of error in the PL ratio method is the band filling effect, which occurs at high excitation powers. Notably, the results of both methods indicate that at low excitation powers, hot carrier effects are weak $(\Delta T \rightarrow 0)$. By increasing the excitation power, there is an increase in $\Delta T$, which is indicative of stronger hot carrier effects in the system.

\section{EXPERIMENTAL RESULTS AND DISCUSSIONS}

The spatial distribution of hot carriers in the MQW structure is investigated via hyperspectral luminescence imaging. With 3D images provided by this technique, it is possible to have access to the PL spectra emitted by different regions of the sample, and by analyzing these spectra, one can study hot carrier effects across the sample. In addition, due to the large collection area of the hyperspectral imager, the effects of local laser excitation and charge diffusion in the planar structure can be investigated. Therefore, it is possible to study the effects of hot carriers due to the local laser excitation, similar to a power-dependent experiment, in a single measurement.

Figure 5(a) illustrates the PL spectra emitted by the InGaAs MQW structure at various lattice temperatures (from $80 \mathrm{~K}$ to $300 \mathrm{~K}$ ) excited by the $405 \mathrm{~nm}$ laser diode with a power density of $630 \mathrm{~W} / \mathrm{cm}^{2}$. At elevated lattice temperatures, the PL peak position shifts to lower energies due to the expansion of the crystal lattice. ${ }^{42}$ In addition, there is an increase in the PL linewidth broadening as a function of lattice temperature. This behavior is attributed to a combination of the occupation of excited states and enhanced hot carrier effects in the MQW structure. ${ }^{31,43}$

Figure 5(b) shows the distribution of $\Delta T(\mathrm{x})$ determined from the PL ratio method as a function of distance from the center of 

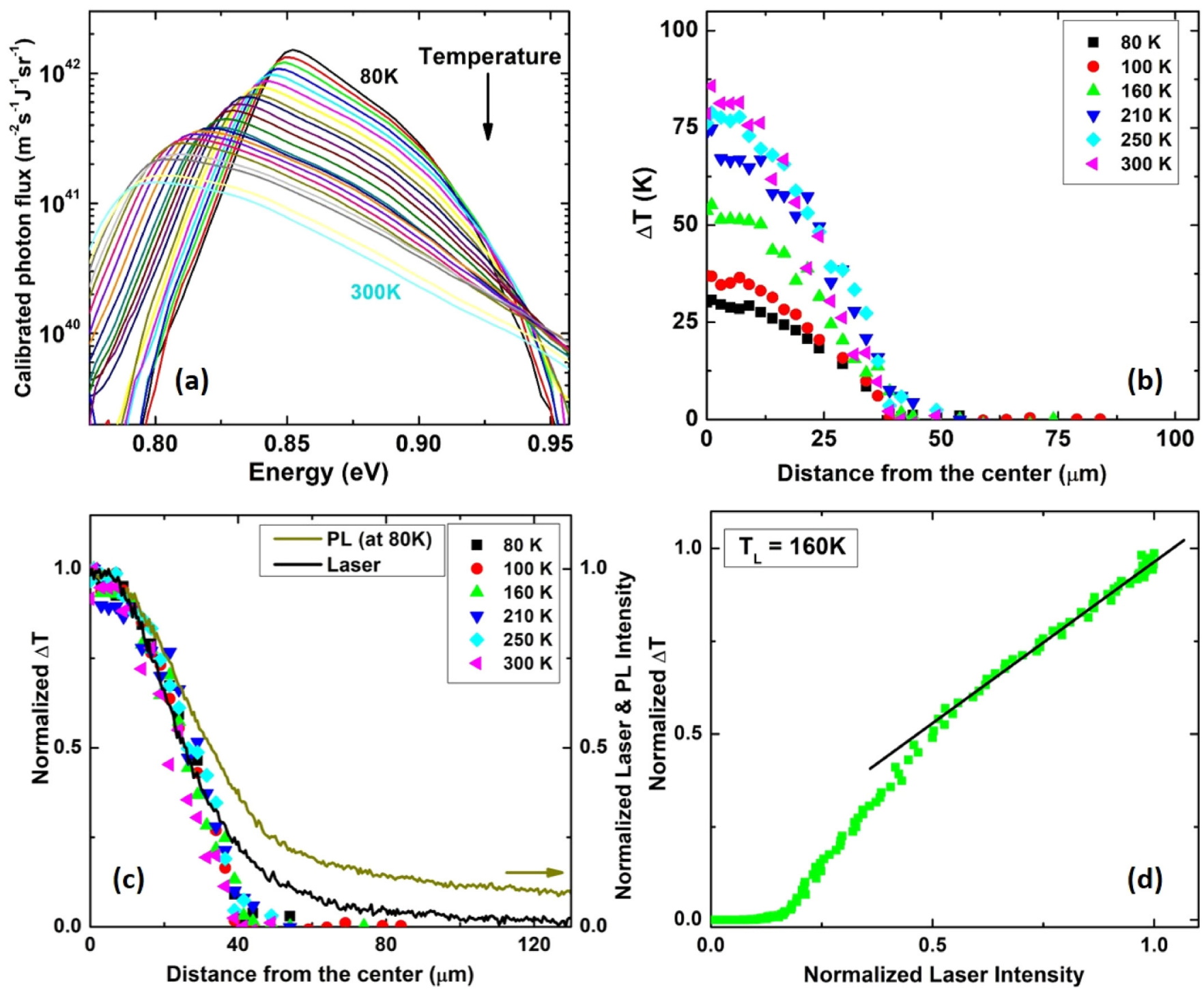

FIG. 5. (a) Temperature-dependent PL spectra emitted from the center of the laser spot. (b) $\Delta T$ vs distance at various lattice temperatures. (c) Normalized $\Delta T$ (colorful symbols) and normalized intensity [laser (black line) and PL (dark green line)] as a function of distance from the laser spot. (d) Normalized $\Delta T$ at $160 \mathrm{~K}$ vs normalized laser intensity.

the laser spot. It is observed that $\Delta T$ is maximized at the center $(\Delta x=0 \mu \mathrm{m})$, and then it decreases at larger distances. In addition, it is seen that at around $\sim 40 \mu \mathrm{m}$ from the center of excitation, $\Delta T$ becomes zero, indicating that photo-generated carriers are completely thermalized at this distance.

Figure 5(b) indicates that there is an increase in $\Delta T$ as a function of lattice temperature, which is significant for hot carrier solar cell applications. The origin of this unusual behavior in the InGaAs MQW structure is still under investigation. In semiconductors, the density of phonons increases with the lattice temperature; therefore, thermalization of hot carriers via phonon-mediated channels becomes more probable. In other words, $\Delta T$ is expected to be smaller at elevated lattice temperatures.

In Fig. 5(c), the normalized $\Delta T$ with respect to the maximum values at each lattice temperature is plotted as a function of the distance from the laser spot. In addition, the normalized spatial distributions of the laser (black) and the PL emission (dark green) at $80 \mathrm{~K}$ are plotted in Fig. 5(c). Due to the similarity among the spatial distribution of PL emission at various lattice temperatures, the one at $80 \mathrm{~K}$ is shown in Fig. 5(c). The laser distribution is Gaussian with the intensity at the center of $354.5 \mathrm{~W} / \mathrm{cm}^{2}$ and the full width at half maximum (FWHM) of $50 \mu \mathrm{m}$. 
Figure $5(\mathrm{c})$ indicates that $\Delta T(\mathrm{x})$ is proportional to the local excitation intensity " $I(x)$." It follows the spatial distribution of the laser profile, rather than the PL distribution, up to a certain distance, and then there is a discrepancy. In addition, Fig. 5(c) shows that at large distances from the center $(\Delta x>90 \mu \mathrm{m})$ where the local laser intensity is negligible, there is still a considerable radiative recombination intensity by photo-generated carriers. This effect is associated with the diffusion of carriers in the planar structure due to the gradient in the charge density, which makes them move and recombine at a position different from where they are generated. ${ }^{44,45}$

As shown in Fig. 5(c), the change in $\Delta T$ as a function of distance follows the spatial distribution of the laser up to $\sim 35 \mu \mathrm{m}$; beyond, the magnitude of $\Delta T$ quickly drops to zero. This effect indicates that above $(\Delta x>35 \mu \mathrm{m})$ the local laser intensity is not sufficient to create a stabilized hot carrier population in the MQW structure. Therefore, it is possible to determine the threshold power $\left(P_{\text {threshold }}=95 \mathrm{~W} / \mathrm{cm}^{2}\right)$ for the generation of non-equilibrium hot carriers in the sample.

The relationship between the normalized $\Delta T$ and the normalized laser intensity is shown in Fig. 5(d). Due to the similarity in the FWHM of the spatial distribution of the normalized $\Delta T$ at various lattice temperatures, the results at $160 \mathrm{~K}$ are plotted in Fig. 5 (d). It is seen that up to about $15 \%$ of the total power of the laser, no significant distribution of hot carriers exists in the system $(\Delta T=0)$. This effect is attributed to an insufficient laser intensity for the generation of hot carriers in the MQW structure. By increasing the local laser intensity (or moving toward the laser spot), evidence of hot carrier effects is observed in the sample. As shown in Fig. 5(d), there is a linear dependence between $\Delta T$ and the laser intensity, especially at higher local intensities (normalized intensity $>0.5$ ). However, it is seen that the variation of $\Delta T$ as a function of intensity decreases at higher local illuminations. This effect can be attributed to energy dissipation by hot carrier transport from the center of the laser spot, with a high carrier density, to surrounding areas with lower densities and temperatures. ${ }^{41}$ As a result, the local density of photo-generated hot carriers decreases, which can reduce the average temperature of carriers near the center spot. ${ }^{6}$ The effects of the size of the laser spot on the hot carrier diffusion and the thermalization coefficient in the MQW structure will be discussed elsewhere.

\section{CONCLUSIONS}

In conclusion, different fitting methods are presented to determine the temperature of carriers from the PL spectrum. It is shown that the extraction of the carrier temperature by the linear fitting can lead to inaccurate results, especially for nanostructured materials. We discuss the conditions under which the PL ratio method can be applied, as an alternative approach, instead of the full PL spectrum fitting, which is a more accurate but more complicated technique.

The spatial distribution of hot carriers in the InGaAs MQW structure is investigated at various lattice temperatures. It is observed that there is an increase in $\Delta T$ as a function of lattice temperature, which indicates that at elevated temperatures, the thermalization of hot carriers is not the dominant mechanism in the system. In addition, a linear dependence between $\Delta T(x)$ and the local laser intensity is seen above the threshold power. Moreover, it is observed that $\Delta T(x)$ reduces at larger distances from the laser spot, and above $\Delta x>40 \mu \mathrm{m}$, no evidence of a hot carrier distribution exists in the MQW structure. Finally, it is seen that at very large distances from the concentrated laser $(\Delta x>90 \mu \mathrm{m})$ where the level of illumination is low, there is still considerable PL emission (or radiative recombination) by the sample. This effect is attributed to the diffusion of photo-generated carriers in the planar structure due to the gradient in the charge density.

\section{ACKNOWLEDGMENTS}

This study is carried out within the framework of program 6: PROOF (Proof of concept for PV innovation breakthrough) at IPVF. The authors would like to acknowledge the Laboratory Foton INSA in Rennes, France, for providing the MQW structure and the LIA NextPV currently IRP NextPV-II, a CNRS-U Tokyo collaboration. The authors acknowledge financial support through the French ANR project ICEMAN (ANR-19-CE05-0019). It was also supported by 3rd Programme d'Investissements d'Avenir (ANR-18EUR-0006-02), and by the Foundation of Ecole polytechnique (Chaire "Developpements Technologiques pour une Energie Responsable" and Chaire "Énergies Durables"). This work benefited from the Energy4Climate Interdisciplinary Center (E4C) of IP Paris and Ecole des Ponts ParisTech.

\section{DATA AVAILABILITY}

The data that support the findings of this study are available from the corresponding author upon reasonable request.

\section{REFERENCES}

${ }^{1}$ R. T. Ross and A. J. Nozik, "Efficiency of hot-carrier solar energy converters," J. Appl. Phys. 53, 3813-3818 (1982).

2. Shah, "Hot carriers in quasi-2-D polar semiconductors," IEEE J. Quantum Electron. 22, 1728-1743 (1986).

${ }^{3}$ S. Lyon, "Spectroscopy of hot carriers in semiconductors," J. Lumin. 35, 121-154 (1986).

${ }^{4}$ G. Conibeer, N. Ekins-Daukes, J.-F. Guillemoles, D. Kőnig, E.-C. Cho, C.-W. Jiang, S. Shrestha, and M. Green, "Progress on hot carrier cells," Solar Energy Mater. Solar Cells 93, 713-719 (2009).

${ }^{5}$ D. König, K. Casalenuovo, Y. Takeda, G. Conibeer, J. Guillemoles, R. Patterson, L. Huang, and M. Green, "Hot carrier solar cells: Principles, materials and design," Physica E Low Dimens. Syst. Nanostruct. 42, 2862-2866 (2010).

${ }^{6}$ Y. Rosenwaks, M. Hanna, D. Levi, D. Szmyd, R. Ahrenkiel, and A. Nozik, "Hot-carrier cooling in GaAs: Quantum wells versus bulk," Phys. Rev. B 48, 14675 (1993)

${ }^{7}$ A. Le Bris, L. Lombez, S. Laribi, G. Boissier, P. Christol, and J.-F. Guillemoles, "Thermalisation rate study of GaSb-based heterostructures by continuous wave photoluminescence and their potential as hot carrier solar cell absorbers," Energy Environ. Sci. 5, 6225-6232 (2012).

${ }^{8}$ D.-T. Nguyen, L. Lombez, F. Gibelli, S. Boyer-Richard, A. Le Corre, O. Durand, and J.-F. Guillemoles, "Quantitative experimental assessment of hot carrierenhanced solar cells at room temperature," Nat. Energy 3, 236-242 (2018).

${ }^{9}$ L. C. Hirst, H. Fujii, Y. Wang, M. Sugiyama, and N. J. Ekins-Daukes, "Hot carriers in quantum wells for photovoltaic efficiency enhancement," IEEE J. Photovoltaics 4, 244-252 (2013).

${ }^{10}$ H. Esmaielpour, V. R. Whiteside, H. P. Piyathilaka, S. Vijeyaragunathan, B. Wang, E. Adcock-Smith, K. P. Roberts, T. D. Mishima, M. B. Santos, 
A. D. Bristow et al., "Enhanced hot electron lifetimes in quantum wells with inhibited phonon coupling," Sci. Rep. 8, 12473 (2018).

${ }^{11}$ G. Conibeer, S. Shrestha, S. Huang, R. Patterson, H. Xia, Y. Feng, P. Zhang, N. Gupta, M. Tayebjee, S. Smyth et al., "Hot carrier solar cell absorber prerequisites and candidate material systems," Solar Energy Mater. Solar Cells 135, 124-129 (2015).

${ }^{12}$ H. Levard, S. Laribi, and J.-F. Guillemoles, "Phonon lifetime in SiSn and its suitability for hot-carrier solar cells,” Appl. Phys. Lett. 104, 222106 (2014).

${ }^{13}$ D. König, Y. Yao, B. Puthen-Veettil, and J. Smith, "Non-equilibrium dynamics, materials and structures for hot carrier solar cells: A detailed review," Semicond. Sci. Technol. 35, 073002 (2020).

${ }^{14}$ D. Ferry, "In search of a true hot carrier solar cell," Semicond. Sci. Technol. 34, 044001 (2019)

${ }^{15}$ H. Esmaielpour, K. R. Dorman, D. K. Ferry, T. D. Mishima, M. B. Santos, V. R. Whiteside, and I. R. Sellers, "Exploiting intervalley scattering to harness hot carriers in III-V solar cells," Nat. Energy 5, 336-343 (2020).

${ }^{16} \mathrm{~J}$. Shah, Ultrafast Spectroscopy of Semiconductors and Semiconductor Nanostructures (Springer Science \& Business Media, 2013), Vol. 115.

${ }^{17}$ Y. Yang, D. P. Ostrowski, R. M. France, K. Zhu, J. Van De Lagemaat, J. M. Luther, and M. C. Beard, "Observation of a hot-phonon bottleneck in lead-iodide perovskites," Nat. Photonics 10, 53-59 (2016).

${ }^{18} \mathrm{M}$. Li, S. Bhaumik, T. W. Goh, M. S. Kumar, N. Yantara, M. Grätzel, S. Mhaisalkar, N. Mathews, and T. C. Sum, "Slow cooling and highly efficient extraction of hot carriers in colloidal perovskite nanocrystals," Nat. Commun. 8, 1 (2017).

${ }^{19}$ J. C. Johannsen, S. Ulstrup, F. Cilento, A. Crepaldi, M. Zacchigna, C. Cacho, I. E. Turcu, E. Springate, F. Fromm, C. Raidel et al., "Direct view of hot carrier dynamics in graphene," Phys. Rev. Lett. 111, 027403 (2013).

${ }^{20}$ J. F. Sierra, I. Neumann, M. V. Costache, and S. O. Valenzuela, "Hot-carrier Seebeck effect: Diffusion and remote detection of hot carriers in graphene," Nano Lett. 15, 4000-4005 (2015)

${ }^{21}$ A. El Fatimy, R. L. Myers-Ward, A. K. Boyd, K. M. Daniels, D. K. Gaskill, and P. Barbara, "Epitaxial graphene quantum dots for high-performance terahertz bolometers," Nat. Nanotechnol. 11, 335-338 (2016).

$\mathbf{2 2}_{\mathrm{J}}$. Shah and R. Leite, "Radiative recombination from photoexcited hot carriers in GaAs,” Phys. Rev. Lett. 22, 1304 (1969).

${ }^{23} \mathrm{G}$. Lasher and F. Stern, "Spontaneous and stimulated recombination radiation in semiconductors," Phys. Rev. 133, A553 (1964).

${ }^{24}$ P. Wurfel, "The chemical potential of radiation," J. Phys. C: Solid State Phys. 15, 3967 (1982)

${ }^{25}$ J. Rodière, L. Lombez, A. Le Corre, O. Durand, and J.-F. Guillemoles, "Experimental evidence of hot carriers solar cell operation in multi-quantum wells heterostructures," Appl. Phys. Lett. 106, 183901 (2015).

${ }^{\mathbf{2 6}} \mathrm{F}$. Gibelli, L. Lombez, and J.-F. Guillemoles, "Two carrier temperatures nonequilibrium generalized Planck law for semiconductors," Phys. B: Condens. Matter 498, 7-14 (2016).

${ }^{27} \mathrm{H}$. Esmaielpour, M. Giteau, A. Delamarre, F. Gibelli, D.-T. Nguyen, N. Cavassilas, Y. Okada, J.-F. Guillemoles, L. Lombez, and D. Suchet, "Advanced analysis for hot-carriers photoluminescence spectrum," Proc. SPIE 11275, 112750A (2020).
${ }^{28}$ J. A. Nelson, The Physics of Solar Cells (World Scientific Publishing Company, 2003).

${ }^{29} \mathrm{P}$. Würfel and U. Würfel, Physics of solar cells: from basic principles to advanced concepts (John Wiley \& Sons, 2016).

${ }^{30}$ F. Gibelli, L. Lombez, and J.-F. Guillemoles, "Accurate radiation temperature and chemical potential from quantitative photoluminescence analysis of hot carrier populations," J. Phys.: Condens. Matter 29, 06LT02 (2016).

${ }^{31}$ H. Esmaielpour, V. R. Whiteside, L. C. Hirst, J. G. Tischler, C. T. Ellis, M. P. Lumb, D. V. Forbes, R. J. Walters, and I. R. Sellers, "Effect of occupation of the excited states and phonon broadening on the determination of the hot carrier temperature from continuous wave photoluminescence in InGaAsP quantum well absorbers," Prog. Photovolt. Res. Appl. 25, 782-790 (2017).

${ }^{32}$ J. Shah, R. Leheny, and C. Lin, "Dynamic Burstein shift in GaAs," Solid State Commun. 18, 1035-1037 (1976).

${ }^{33}$ D. Erskine, A. Taylor, and C. Tang, "Dynamic Burstein-Moss shift in GaAs and GaAs/AlGaAs multiple quantum well structures," Appl. Phys. Lett. 45, 1209-1211 (1984).

${ }^{34}$ E. Burstein, "Anomalous optical absorption limit in InSb," Phys. Rev. 93, 632 (1954).

${ }^{35}$ T. Moss, "The interpretation of the properties of indium antimonide," Proc. Phys. Soc. B 67, 775 (1954).

36. Blakemore, "Approximations for Fermi-Dirac integrals, especially the function $\mathrm{F}_{12}(\eta)$ used to describe electron density in a semiconductor," Solid State Electron. 25, 1067-1076 (1982).

${ }^{37}$ D. Chemla, D. Miller, P. Smith, A. Gossard, and W. Wiegmann, "Room temperature excitonic nonlinear absorption and refraction in GaAs/AlGaAs multiple quantum well structures," IEEE J. Quantum Electron. 20, 265-275 (1984).

${ }^{38} \mathrm{M}$. Colocci, M. Gurioli, and A. Vinattieri, "Thermal ionization of excitons in GaAs/AlGaAs quantum well structures,” J. Appl. Phys. 68, 2809-2812 (1990).

${ }^{39}$ Y. Chen, R. Cingolani, L. Andreani, F. Bassani, and J. Massies, "Photoluminescence in quantum well and bulk GaAs: A direct comparative study," Il Nuovo Cimento D 10, 847-859 (1988).

${ }^{40}$ R. N. Zitter, "Saturated optical absorption through band filling in semiconductors," Appl. Phys. Lett. 14, 73-74 (1969).

${ }^{41} \mathrm{P}$. Yuan, J. Liu, R. Wang, and X. Wang, "The hot carrier diffusion coefficient of sub-10 nm virgin $\mathrm{MoS}_{2}$ : Uncovered by non-contact optical probing," Nanoscale 9, 6808-6820 (2017).

${ }^{42}$ Y. P. Varshni, "Temperature dependence of the energy gap in semiconductors," Physica 34, 149-154 (1967).

${ }^{43}$ J. Lee, E. S. Koteles, and M. Vassell, "Luminescence linewidths of excitons in GaAs quantum wells below 150 k,” Phys. Rev. B 33, 5512 (1986).

${ }^{44} \mathrm{U}$. Jahn, T. Flissikowski, H. Grahn, R. Hey, E. Wiebicke, A. Bluhm, J. Miguel-Sánchez, and A. Guzmán, "Carrier diffusion lengths of (In, Ga) As, GaAs and (In, Aa) (As, N) quantum wells studied by spatially resolved cathodoluminescence," in Microscopy of Semiconducting Materials (Springer, 2005), pp. $467-470$

${ }^{45}$ A. W. Walker and M. W. Denhoff, "Heavy and light hole minority carrier transport properties in low-doped n-InGaAs lattice matched to InP," Appl. Phys. Lett. 111, 162107 (2017). 